\title{
Commentary
}

\section{Contact Dermatitis from Hand Hygiene Practices in the COVID-19 Pandemic}

See Wei Tan, ${ }^{1} M B B S, M R C P$, Choon Chiat $\underline{\mathrm{Oh}},{ }^{1}$ MBBS, FRCP, FAMS (Dermatology)

\begin{abstract}
Coronavirus disease 2019 (COVID-19) pandemic continues to spread globally at a staggering speed. At present, there is no effective treatment or vaccine for COVID-19. Hand disinfection is a cost-effective way to prevent its transmission. According to the Centres for Disease Control and Prevention (CDC) guidelines, we should wash our hands with soap and water for at least 20 seconds. If soap and water are not readily available, alcohol-based hand rubs (ABHRs) with at least $60 \%$ alcohol are the alternative. With diligent hand disinfection reinforced during COVID-19, there is an increased prevalence of contact dermatitis. This commentary highlights the fact that contact dermatitis is a readily treatable condi tion and should not cause any deviation of proper hand hygiene. In irritant contact dermatitis (ICD), the management strategies are selection of less irritating hand hygiene products, frequent use of moisturisers to rebuild the skin barrier, and education on proper hand hygiene practices. In allergic contact dermatitis (ACD), the identification and avoidance of the contact allergen is the key to treatment. However, ACD is less common and only accounts for $20 \%$ of the cases. The identified allergens in hand cleansers are predominantly preservative excipients a nd ACD attributable to ABHR are very uncommon. Alcohol-free hand rubs are widely available on the market but it is not a recommended alternative to ABHRs by the CDC.
\end{abstract}

Ann Acad Med Singap. 2020;49:674-76

Coronavirus disease 2019 (COVID-19) was declared a pandemic by the World Health Organization (WHO) on 11 March 2020. This is the first coronavirus pandemic in human history. The spread of this on-going pandemic is outpacing the capacity and resources of the health care system worldwide. The age-adjusted case fatality rate of COVID-19 is $0.5 \%$, which is 5 times the commonly cited case fatality rate of adult seasonal influenza. ${ }^{1}$

COVID-19 is caused by the severe acute respiratory syndrome coronavirus 2 (SARS-CoV-2). The human-tohuman transmission of SARS-CoV-2 can occur by direct contact with infected people via respiratory droplets and indirectly via contact with contaminated surfaces.
SARS-COV-2 was found to be viable and remained infectious on surfaces for up to 72 hours. ${ }^{2}$ This finding highlights the necessity of practising good hand hygiene and the importance of regular disinfection of commonly touched surfaces, such as doorknobs, toilet handles and escalator handrails.

At present, there is no effective treatment or vaccine for COVID-19. Countries have introduced various recommendations to prevent transmission of COVID-19, and one cost-effective way is hand disinfection. According to the Centres for Disease Control and Prevention (CDC) guidelines, we should wash our hands with soap and water for at least 20 seconds. This step is especially

\footnotetext{
${ }^{1}$ Department of Dermatology, Singapore General Hospital, Singapore

Address for Correspondence: Dr Oh Choon Chiat, Department of Dermatology, Singapore General Hospital, 20 College Road, Academia, Singapore 169856 Email: oh.choon.chiat@singhealth,com.sg
} 
important before eating, after going to the toilet, blowing your nose, coughing and sneezing. If soap and water are not readily available, alcohol-based hand rubs (ABHRs) with at least $60 \%$ alcohol are endorsed by the $\mathrm{CDC}$ as an effective method of hand hygiene practice. ${ }^{3}$

ABHRs use ethanol, isopropanol, and n-propanol as antimicrobial agents. Alcohol denatures proteins and has good activity against enveloped viruses, including coronaviruses. ${ }^{4}$ To be effective against microorganisms, at least $3 \mathrm{~mL}$ of the hand rub is to be applied to dry hands, and rubbing should last for at least 10 to 30 seconds until the hands are dry. Proper application of ABHRs with the 6-step hand rub technique as recommended by the WHO has the same antimicrobial efficacy as hand washing with detergents. ${ }^{4}$ Compared to hand washing, ABHRs are a more readily accessible and are a convenient alternative. In Singapore hospitals, ABHRs are readily available for health care workers at the bedside of every patient. For the general public, ABHRs are also widely available in small bottles for pocket carriage. Hand rubs require less time to perform than hand washing. The convenient and on-the-go method of cleaning your hands with ABHRs has made it a more attractive hand-disinfecting method for most people.

Contact dermatitis is very common among health care workers who are required to perform frequent hand disinfection and therefore are repeatedly expososed to irritants in hand hygiene products. With diligent hand disinfection reinforced during COVID-19, there is an increased prevalence of contact dermatitis. ${ }^{5}$ Contact dermatitis from hand hygiene products is an important barrier to compliance with hand hygiene. ${ }^{6}$ Lapses in proper hand hygiene can increase the risk of contracting and transmitting COVID-19. This challenge can be overcome as contact dermatitis is a readily treatable condition. The emphasis is on proper hand hygiene and regular use of skin moisturisers.

Contact dermatitis may either be irritant induced, causing irritant contact dermatitis (ICD), or allergen induced, causing allergic contact dermatitis (ACD). ICD is more common and accounts for nearly $80 \%$ of cases. ${ }^{7}$ Repeated exposure to ABHRs can induce pathophysiologic changes, such as denaturation of the stratum corneum proteins, changes in intercellular lipid moieties, and impairment of keratinocytes, which leads to the subsequent release of pro-inflammatory cytokines. However, the reactions of the stratum corneum and keratinocytes vary with the alcohol type. A study showed that isopropanol and n-propanol caused significant stratum corneum and keratinocyte perturbations, whereas ethanol did not. Therefore, ethanol-based sanitizers are better tolerated by the skin. ${ }^{8}$ The antiseptic ingredients used in hand wash products, such as chlorhexidine and triclosan, are common skin irritants. Frequent hand washing strips off the natural oils of the hands, facilitating penetration of the irritants through the disrupted epidermal barrier and initiation of inflammatory cascades.

ACD is uncommon and occurs in only $20 \%$ of cases. ACD is a type IV, T cell-mediated, delayed hypersensitivity reaction to an allergen. The first exposure to the allergen causes sensitization of the inflammatory cells in the body. Upon re-exposure to the allergen, the sensitized $\mathrm{T}$ cells will initiate inflammatory cascades leading to the eruption of the ACD. The eruption usually starts 48 hours or days after contact with the allergens. Repeated exposures can lead to crescendo reactions.

Differentiating ACD and ICD is critical because the approach and management strategies are different. In ICD, the predominant clinical features include burning, stinging, and soreness of the skin. Clinical features of ACD are predominantly characterised by pruritus. Irritant reactions in ICD are characterised by the decrescendo phenomenon where the elicitation time is short, reaches its peak within minutes to hours, and then starts to heal. Lesions in ACD usually appear 24 to 48 hours after the last exposure to the causative agent and exhibit a crescendo phenomenon by reaching their peak reaction at 72 to 96 hours. The eruption of ICD is commonly limited to the area of exposure, whereas secondary spread may occur in ACD. Although there are distinguishing features between ACD and ICD, it is difficult clinically to differentiate ICD and ACD unambiguously. ${ }^{7}$ The skin patch test helps diagnose allergic reactions in ACD.

In ICD, the management approaches are selection of less irritating hand hygiene products, frequent use of moisturisers to rebuild the skin barrier and education on proper hand hygiene practices. Among the commonly used hand disinfectants, ABHRs have the lowest irritant potential compared to that of triclosan, chlorhexidine and quaternary ammonium compounds. ${ }^{5,9}$ To further minimise the risk of skin irritation, most of the commercially available ABHR formulations have added humectants that are proven to reduce contact dermatitis without compromising the antiseptic efficacy. Although various Singapore hospitals use different brands of ABHRS, all of them have emollient added into the formulation. One of the widely used ABHRs in Singapore hospitals, "Softa-Man", has PEG-6 caprylic/capric glycerides added as the emollient.

Hand moisturisers contain humectants, fats and oils that can effectively increase skin hydration and replace depleted skin lipids, which help to restore the barrier function of the skin. ${ }^{10}$ There are several types of 
moisturisers available on the market. Humectants, namely, topical urea and propylene glycol, are capable of attracting water to the stratum corneum from the environment and the deeper layers of the skin. ${ }^{10}$

Occlusive emollients, such as petrolatum-based products, mineral oils, and waxes, can prevent water loss and alleviate irritation. A combination of these two is useful for attracting and sealing water at the stratum corneum layer and soothing the skin. The moisturisers are effective only when present on the skin. Therefore, they should be liberally applied multiple times a day, particularly after hand washing and ABHR application, to support the regenerative capacities of the skin. The effectiveness of ABHRs is not reduced by prior use of hand moisturisers. ${ }^{10}$ To reduce the risk of contact dermatitis, it is highly recommended to use fragrance-free and hypoallergenic products.

Washing hands with soap and water alone is adequate and can be effective, but may also be a cause of irritant contact dermatitis. Certain common mistakes in hand hygiene practices can increase the risk of skin irritation. For example, washing hands with soap and water immediately before or after using an alcohol-based product is unnecessary and will lead to dermatitis. Hand washing can remove the natural oils and emollients from the skin. Application of ABHRs on the skin after hand washing could induce a burning sensation, as the alcohol can readily penetrate the sensory nerve endings in the pre-irritated skin. This phenomenon creates a public misconception of ABHRs as the culprit irritant instead of the underlying disrupted epidermal barrier caused by hand washing. Hence, it is important to adopt proper skin disinfection practices to reduce contact dermatitis.

In $\mathrm{ACD}$, the identification and avoidance of the contact allergen is the key to treatment. The culprit allergen can usually be identified by performing a skin patch test. Higgins et al. conducted a study among health care workers for 21 years and identified the predominant allergens in hand cleansers to be preservative excipients, such as coconut diethanolamide $(5.3 \%)$, chlorhexidine diacetate $(2.2 \%)$, and chlorhexidine digluconate $(2.2 \%){ }^{9}$ It is important to note that ACD attributable to ABHRs is very uncommon. However, if the identified culprit allergen is alcohol, patients should stop using ABHRs.

Alcohol-free hand rubs are widely available on the market, and quaternary ammonium compounds (QACs) are one of the most popular disinfectants used in alcoholfree hand rubs. In general, QACs are relatively well tolerated and have low allergenic potential. However, it is not a recommended alternative to ABHRs by the CDC because QACs have less reliable activity against viruses than alcohol. ${ }^{3}$ In conclusion, given the soaring infection rates of COVID-19, compliance with hand hygiene recommendations should not be compromised by contact dermatitis. The potential development of ICD is preventable and manageable by the adoption of proper hand care routines. Therefore, we should not deviate from the strict hand hygiene rules. Proper hand hygiene practices, regular application of moisturisers, and avoidance of the known culprit allergen are the key components in the prevention of contact dermatitis from hand hygiene products.

\section{REFERENCES:}

1. Faust JS, Del Rio C. Assessment of Deaths From COVID-19 and From Seasonal Influenza. JAMA Intern Med 2020 May 14th; Epub Ahead of Print.

2. Shi AH, Guo W, Chng CK, Chan BH. Precautions When Providing Dental Care During Coronavirus Disease 2019 (COVID-19) Pandemic. Ann Acad Med Singap 2020;49:312-319.

3. Coronavirus Disease 2019(COVID-19). 2020; Available at: https://www. cdc.gov/coronavirus/2019-ncov/hcp/hand-hygiene.html. Accessed May 31, 2020.

4. Widmer AF. Replace hand washing with use of a waterless alcohol hand rub? Clin Infect Dis 2000;31:136-143.

5. Elston DM. Occupational skin disease among healthcare workers during the Coronavirus (COVID-19) epidemic. J Am Acad Dermatol 2020;82:1085-1086.

6. Visscher MO, Randall Wickett R. Hand hygiene compliance and irritant dermatitis: a juxtaposition of healthcare issues. Int J Cosmet Sci 2012;34:402-415.

7. Ale SI, Maibach HI. Irritant Contact Dermatitis ersus Allergic Contact Dermatitis. In: Chew AL, Maibach HI. Editors. Irritant Dermatitis. Berlin, Heidelberg. Springer 2006.

8. Cartner T, Brand N, Tian K, Saud A, Carr T, Stapleton P, et al. Effect of different alcohols on stratum corneum kallikrein 5 and phospholipase A2 together with epidermal keratinocytes and skin irritation. Int J Cosmet Sci 2017;39:188-196.

9. Higgins CL, Palmer AM, Cahill JL, Nixon RL. Occupational skin disease among Australian healthcare workers: a retrospective analysis from an occupational dermatology clinic, 1993-2014. Contact Dermatitis 2016;75:213-222.

10. Paula H, Hübner N, Assadian O, Bransmöller K, Baguhl R, Löffler $\mathrm{H}$, et al. Effect of hand lotion on the effectiveness of hygienic hand antisepsis: Implications for practicing hand hygiene. Am J Infect Control 2017;45:835-838. 